\title{
The QUEENS experiment through TEFIS platform
}

\author{
G. Aristomenopoulos ${ }^{1}$, A. Kaninis ${ }^{2}$, P. Vlahopoulos ${ }^{2}$, A. Sällström ${ }^{3}$, F. Benbadis ${ }^{4}$, \\ S. Papavassiliou ${ }^{1}$ \\ ${ }^{1}$ Institute of Communications and Computer Systems, Greece, ${ }^{2}$ VELTI SA of Software \\ Products \& Related Products \& Services, Greece, ${ }^{3}$ Lulea University of Technology - Centre \\ for Distance-spanning Technology, Sweden, ${ }^{4}$ Thales Communications and Security, France
}

\begin{abstract}
QUEENS (Dynamic Quality User Experience ENabling Mobile Multimedia Services) aims at exploiting and leveraging on the benefits and flexibility provided by a novel dynamic real-time QoE provisioning framework in order to assess and establish a new era of user-centric mobile multimedia applications. To achieve that, QUEENS utilizes and builds on the TEFIS infrastructure (EC ICT FIRE initiative) that provides a unique integrated platform for supporting efficient Future Internet service experiment and development involving different actors and heterogeneous testbeds. The TEFIS tools and facilities for designing, planning and executing QUEENS experiment, along with a QoE-aware mobile multimedia application prototype will be demonstrated, forming the trends of future internet services and experimental procedures.
\end{abstract}

Keywords: Experimental testing Facilities, Quality of Experience, Multimedia Services.

\section{The QUEENS Experiment and TEFIS Platform}

QUEENS (Dynamic Quality User Experience ENabling Mobile Multimedia Services) aims at establishing, assessing, evolving and prototyping a novel framework for extending QoS (Quality of Service) to QoE (Quality of Experience) in mobile wireless networks, placing emphasis on mobile on-demand multimedia applications. Among the key features of the QUEENS framework is the joint consideration of usercentric QoE preferences and network-centric mechanisms to enable a novel crosslayering approach based on Network Utility Maximization (NUM) theory [1], which allows the operation optimization and the resource utilization maximization of: a) mobile application's server and b) underlying wireless network radio resource management processes.

To facilitate its goals the unique characteristics of TEFIS integrated testbed platform [2] is exploited by QUEENS experiment. TEFIS (FIRE Initiative), a TEstbed for Future Internet Services, provides a single point of access to a set of federated testbed resources, exploited by QUEENS towards enabling: a) real-time end-users' QoE provisioning (User Interface) experiments, b) performing large scale mobile emulation networking experiments and, c) validating application prototypes with currently existing standards (i.e., IMS, 3GPP/LTE). 


\section{Background and Demonstration Description}

The TEFIS platform supports the overall experiment lifecycle, by providing a graphical user interface that allows for designing, planning, executing and evaluating the experiment results, eliminating the burden of managing, configuring and operating multiple heterogeneous testbeds, as described in Fig.1. The adoption of this platform for the description and the workflow of QUEENS experiment will be demonstrated.

The initial, part of the QUEENS experiment lays the foundations and the overall concepts of the proposed Dynamic QoE Provisioning mechanism that offers enhanced services to the end users, increased profits to the service and network providers, as well as overall system performance maximization.

An interactive mechanism designed to support enhanced mobile multimedia applications by allowing users to interact with the video currently watched and request for different quality of service towards increasing their QoE will be demonstrated. Specifically, the developed android-based application will be demonstrated that offers a lightweight interactive graphical user interface that allows users to select and rate different video qualities of the same video clip (Fig.2), while providing context information and pricing incentives towards steering their decisions (Fig.3). Testruns by Botnia Living Lab [3] users (as part of the overall TEFIS platform) provide a realistic and pragmatic view of users' requirements and expectations. Moreover, a proficient correlation between QoE, as derived by users' satisfaction rating (Fig.4)) and QoS, by appropriately mapping video file specifications, like codecs, bitrate or fps, to network metrics, like throughput or delay, expressed via the use of proper utility functions, will be illustrated. Finally, pricing incentives that drive users' behavior, as well as its effects on network operators' and service providers' profits will be highlighted.

The derived utility functions correlating QoS to QoE, as well as the knowledge of users' expectations and requirements that will be initially demonstrated, are used as feedback to the next phases. Specifically, regressive and stress tests in large scale environments offered by PlanetLab [4] evaluate the correctness, stability and scalability of the proposed dynamic QoE adaptation scheme in the resource allocation process of the wireless networks and video servers. Moreover, the mobile QoE-aware multimedia application will be prototyped and end-to-end validated via SQS IMS Testbed [5] towards assuring its compliance with the reference protocols and standards, as well as its interoperability on a realistic environment (e.g., 3GPP/LTE).

\section{References}

1. Aristomenopoulos, G., Kastrinogiannis, T., Kaldanis, V., Karantonis, G., Papavassiliou, S.: A Novel Framework for Dynamic Utility-Based QoE Provisioning in Wireless Networks, in Proc. of IEEE Globecom 2010.

2. FP7 EU PROJECT TEFIS (TEstbed for Future Internet Services), http://www.tefisproject.eu/

3. Botnia living labs, http://www.openlivinglabs.eu/node/125

4. PlanetLab Europe, http://www.planet-lab.eu/

5. Software Quality Systems S.A., http://www.sqs.es/en/ 


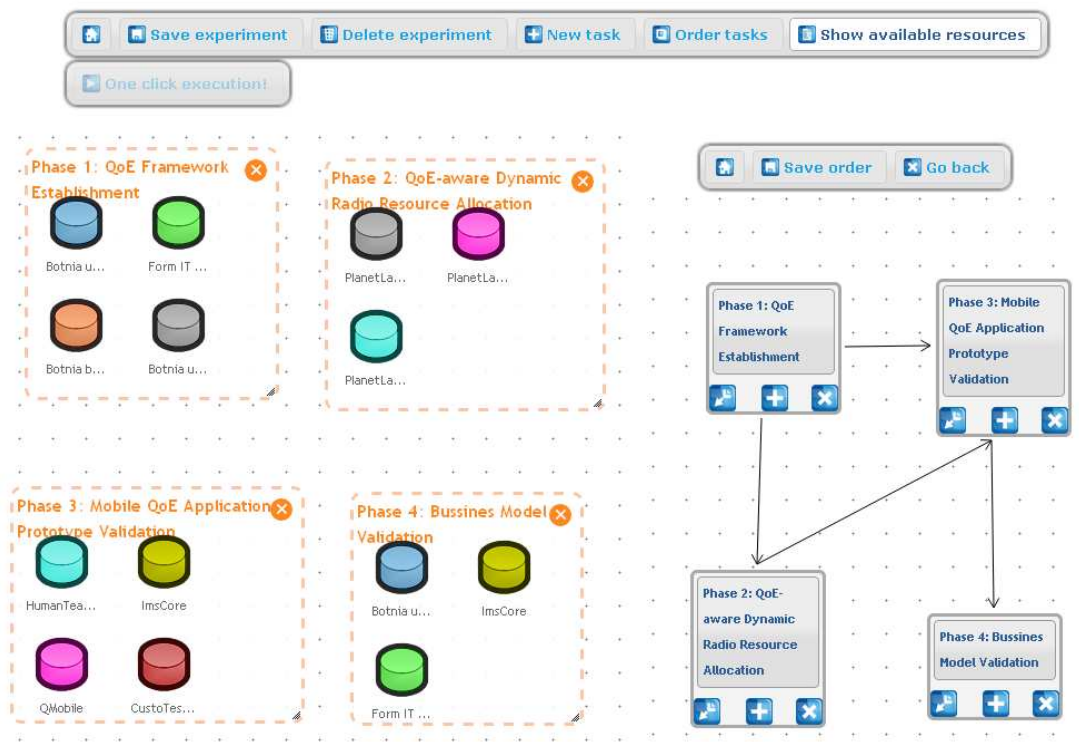

Fig. 1. The QUEENS experiment workflow as described at the TEFIS experiment manager.

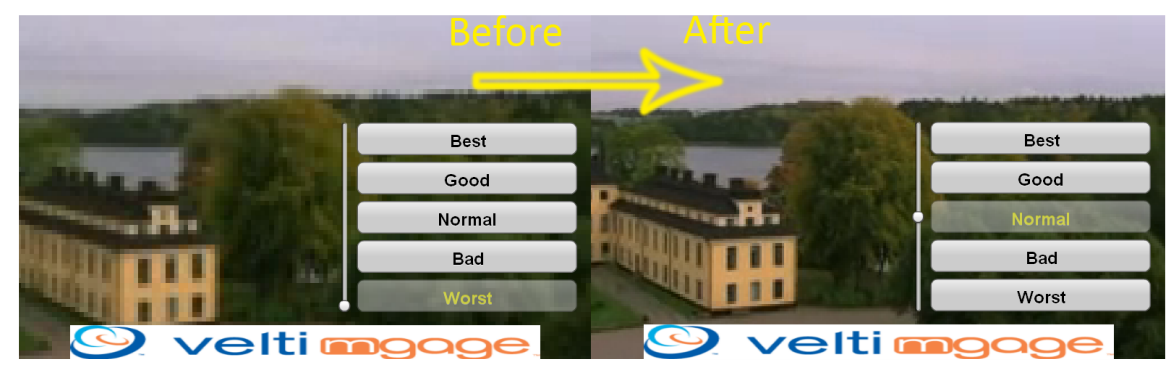

Fig. 2. QoE provisioning graphical user interface (within the mobile multimedia application) 


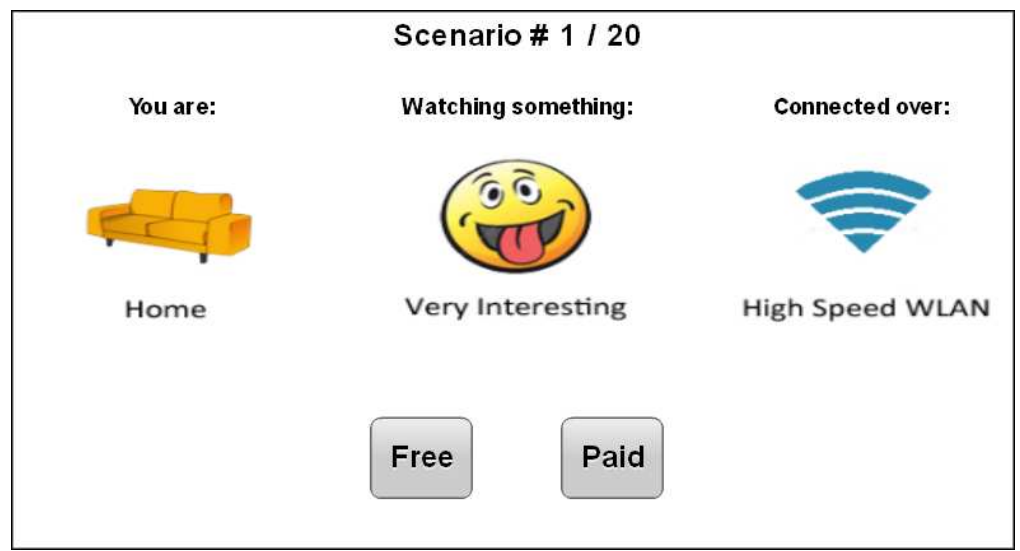

Fig. 3. Context information and pricing incentives

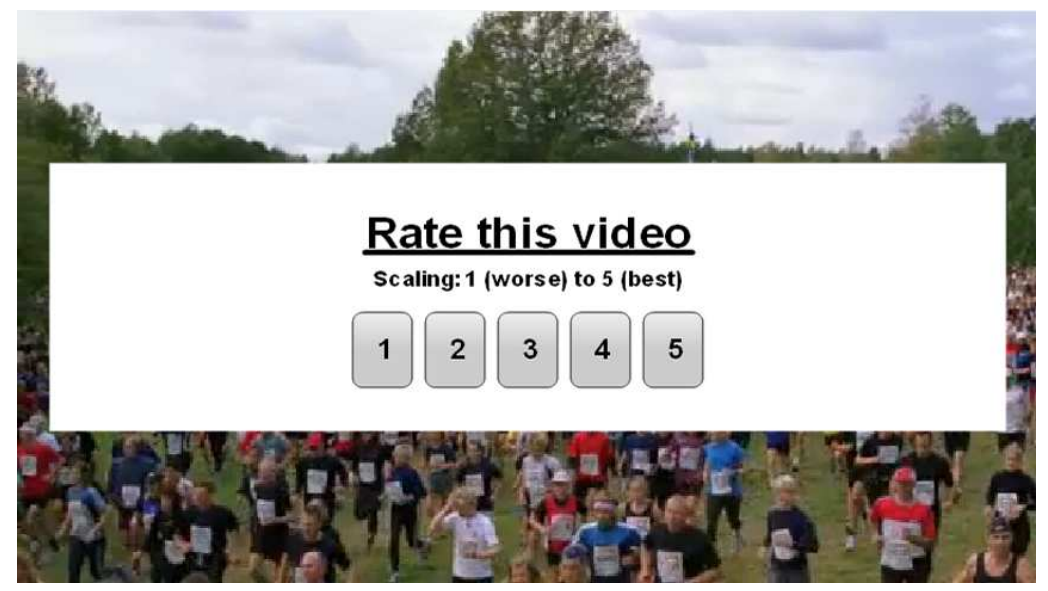

Fig. 4. QoE satisfaction rating 